University of Wollongong

Research Online

Faculty of Engineering and Information

Faculty of Engineering and Information

Sciences - Papers: Part B

Sciences

2018

\title{
Measurement of microsurface topography using a self-mixing optical configuration
}

Xiaohong Sun

Zhengzhou University

Han Wang

University of Wollongong, hw894@uowmail.edu.au

Bin Liu

University of Wollongong, bl987@uowmail.edu.au

Yanguang Yu

University of Wollongong, yanguang@uow.edu.au

Follow this and additional works at: https://ro.uow.edu.au/eispapers1

Part of the Engineering Commons, and the Science and Technology Studies Commons

Research Online is the open access institutional repository for the University of Wollongong. For further information contact the UOW Library: research-pubs@uow.edu.au 


\title{
Measurement of microsurface topography using a self-mixing optical configuration
}

\author{
Abstract \\ This work proposes to measure the topography of microstructure surfaces using a self-mixing \\ interference (SMI) configuration. The theoretical measurement model is built using beam-expanded plane \\ wave method and considering SMI effect. The interference patterns for different objects are obtained \\ based on the presented model. In addition, an algorithm for reconstructing the three-dimensional surface \\ is implemented and applied onto an object with spherical surface. The presented work shows the \\ potential application for topography measurement using a compact SMI configuration.

\section{Keywords} \\ topography, measurement, self-mixing, optical, configuration, microsurface

\section{Disciplines} \\ Engineering | Science and Technology Studies

\section{Publication Details} \\ X. Sun, H. Wang, B. Liu \& Y. Yu, "Measurement of microsurface topography using a self-mixing optical \\ configuration," Optical Engineering, vol. 57, (5) pp. 051503-1-051503-6, 2018.
}




\section{Optical Engineering}

\section{Measurement of microsurface topography using a self-mixing optical configuration}

Xiaohong Sun

Han Wang

Bin Liu

Yanguang $\mathrm{Yu}$ mixing optical configuration," Opt. Eng. 57(5), 051503 (2018), doi: 10.1117/1.OE.57.5.051503. 


\title{
Measurement of microsurface topography using a self-mixing optical configuration
}

\author{
Xiaohong Sun, ${ }^{\mathrm{a}, \mathrm{b}}$ Han Wang, ${ }^{\mathrm{a}}$ Bin Liu, ${ }^{\mathrm{a}}$ and Yanguang $\mathrm{Yu}^{\mathrm{a}, \star}$ \\ aUniversity of Wollongong, School of Electrical, Computer and Telecommunications Engineering, Wollongong, Australia \\ 'Zhengzhou University, School of Information Engineering, Henan Key Laboratory of Laser and Opto-Electric Information Technology, \\ Zhengzhou, China
}

\begin{abstract}
This work proposes to measure the topography of microstructure surfaces using a self-mixing interference (SMI) configuration. The theoretical measurement model is built using beam-expanded plane wave method and considering SMI effect. The interference patterns for different objects are obtained based on the presented model. In addition, an algorithm for reconstructing the three-dimensional surface is implemented and applied onto an object with spherical surface. The presented work shows the potential application for topography measurement using a compact SMI configuration. @ 2018 Society of Photo-Optical Instrumentation Engineers (SPIE) [DOI: 10 .1117/1.0E.57.5.051503]
\end{abstract}

Keywords: microsurface topography; self-mixing interference; optical feedback; three-dimensional reconstruction.

Paper 171772SS received Nov. 6, 2017; accepted for publication Dec. 14, 2017; published online Feb. 8, 2018.

\section{Introduction}

The profile measurement of an object is needed for many applications, such as quality control in a production line, biomedicine, robotics, measurement of mechanical wear, and the recording of surface shape data for the determination of the three-dimensional (3-D) deformation or strain analysis in holographic interferometry. ${ }^{1}$ There are several known methods used for profile measurement: triangulation, fringe projection (so-called moire), interferometric methods, speckle interferometry, and so on. This paper will restrict its attention to interferometric methods.

Compared to the traditional interferometry, self-mixing interferometry, also called optical feedback interferometry, has drawn more and more attention due to its unique features of compactness, low cost, simple implementation, and high sensitivity. An SMI system needs only a laser [normally a laser diode (LD)], a lens, and an external target. Generally, the laser is set at a bias current above its threshold. When the external target exists, a fraction of the light backscattered by the target re-enters the laser cavity. Then, both the frequency and intensity of the emitted laser are modulated. The modulated laser intensity is usually called SMI signal. An SMI signal can be detected by a monitor photodetector packaged in the rear of the LD. The SMI-based applications have been found in various applications of sensing and instrumentation, such as the measurement for absolute distance, displacement, vibration, laser parameters, etc. ${ }^{2-5}$

The SMI-based imaging can be classified into two classes. In one class, the LD is set to operate at a steady state, ${ }^{6-9}$ using SMI in a confocal microscope configuration. The laser is operated at a constant bias current. The optical feedback from a target surface will cause the change in bias current. This change can be directly related to the change in surface profile. The imaging can also be achieved using SMI by linear frequency sweeping. ${ }^{10,11}$ In this case, the bias current is modulated by a triangular function. The optical feedback can cause periodical fluctuation on the triangular waveform. The average frequency variation between the fringes in the increasing and decreasing period of the triangular waveform is proportional to the distance between the laser and the external target. Square-wave modulation scheme is proposed for SMI imaging in THz laser. ${ }^{12}$ This modulation scheme can achieve high frame-rate imaging.

In another class, the laser operates in a relaxation oscillation state. In this case, the dynamical response of a laser is highly sensitive to frequency-shifted optical feedback. Three-dimensional images can be obtained from the amplitude (reflectivity) or phase (profilometry) of a laser output signal by moving the target in three dimensions using micrometric motorized stages or by moving the laser beam using a galvanometric scanner. ${ }^{13,14}$

The resolution of imaging formed in above techniques is determined by the laser beam spot size. To overcome this limit, plenoptic microscope and synthetic aperture methods are explored in works, ${ }^{15-17}$ leading to spatial resolution exceeding the diffraction limit.

However, all the above SMI-based imaging is based on point-by-point scanning, which is time-consuming. In this paper, we present a beam-expanded, SMI for microsurface topography. First, the related theory and simulation are presented for describing the interference pattern obtained at charge-coupled device (CCD) plane. Then the reconstruction algorithm for obtaining 3-D profile of an object is implemented to verify the proposed method.

\section{Theoretical Modeling}

The system schematic diagram is shown in Fig. 1. An elliptical Gaussian beam emitted by LD is beam expanded and collimated into a parallel plane wave. The beam is split into two beams by a 20/80 beam splitter (BS). One beam is called as reference beam indicated by " $R$ " as shown in Fig. 1. The other one is called as object beam indicated by "O" carrying the intensity and phase information of the object. The two beams are interfered on CCD screen, 


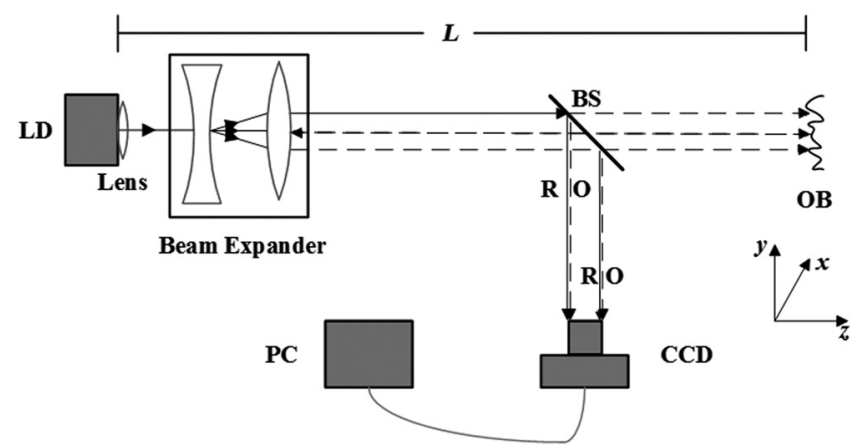

Fig. 1 System schematic: LD, laser diode; BS, beam splitter; OB, object; R, reference beam; O, object beam; CCD, charge-coupled device; and PC, personal computer.

and the interferogram (or called interference pattern) is collected by a personal computer (PC). Further image processing and reconstructing can work out the surface topography for an object.

Considering the plane wave, the complex amplitudes of the $\mathrm{R}$ and $\mathrm{O}$ beams are expressed as follows:

$u_{\mathrm{R}}(x, y, z)=a_{\mathrm{R}} \exp \left(-i \varphi_{\mathrm{R}}\right)$

$u_{\mathrm{O}}(x, y, z)=a_{\mathrm{O}}(x, y) \exp \left[-i \varphi_{\mathrm{O}}(x, y, z)\right]$,

where $a_{\mathrm{R}}$ and $\varphi_{\mathrm{R}}$ are the real amplitude and phase of the $\mathrm{R}$ beam, respectively, and $a_{\mathrm{O}}(x, y)$ and $\varphi_{\mathrm{O}}(x, y, z)$ are the amplitude and phase of the $O$ beam, respectively. So the interference intensity received by CCD is expressed as ${ }^{18}$

$$
\begin{aligned}
I(x, y, z)= & \left|u_{\mathrm{R}}(x, y, z)+u_{\mathrm{O}}(x, y, z)\right|^{2} \\
= & {\left[u_{\mathrm{R}}(x, y, z)+u_{\mathrm{O}}(x, y, z)\right] } \\
& \times\left[u_{\mathrm{R}}(x, y, z)+u_{\mathrm{O}}(x, y, z)\right]^{*} \\
= & a_{\mathrm{R}}^{2}+\left|a_{\mathrm{O}}(x, y)\right|^{2}+2 a_{\mathrm{R}}\left|a_{\mathrm{O}}(x, y)\right| \cos \left(\varphi_{\mathrm{O}}-\varphi_{\mathrm{R}}\right) .
\end{aligned}
$$

Considering the SMI effect, the laser frequency $v_{0}$ will be modified as ${ }^{5}$

$v_{f}=v_{0}-\frac{C}{2 \pi \tau} \sin \left(2 \pi \tau v_{f}+\arctan \alpha\right)$,

where $v_{0}$ and $v_{f}$ are the laser frequency with and without optical feedback respectively, $C$ is the optical feedback level, $\alpha$ is the linewidth-enhancement factor, and $\tau$ is the laser roundtrip time in the external cavity, which is determined by the external cavity length and light speed, expressed by $2[L+z(x, y)] / c$, where $c$ is the light speed. In the following simulations, in this work, we set $C=2, \alpha=3$, $v_{0}=7.41 \times 10^{14} \mathrm{~Hz}$, i.e., $\lambda_{0}=0.405 \mu \mathrm{m}$. In this case, the phase of the $\mathrm{R}$ and $\mathrm{O}$ beams under optical feedback, denoted by $\varphi_{\mathrm{RF}}$ and $\varphi_{\mathrm{OF}}$, can be expressed as

$\varphi_{\mathrm{RF}}=\frac{2 \pi v_{\mathrm{F}} L_{\mathrm{R}}}{c}$

$\varphi_{\mathrm{OF}}=\frac{4 \pi v_{\mathrm{F}}\left[L_{\mathrm{R}} / 2+L+z(x, y)\right]}{c}$,
$\varphi_{\mathrm{OF}}-\varphi_{\mathrm{RF}}=\frac{4 \pi v_{\mathrm{F}}[L+z(x, y)]}{c}$,

where $L_{\mathrm{R}}$ is the distance $\mathrm{R}$ beam traveled and $L$ is the distance between the facet of the LD and the object with $z(x, y)=0$. Then, Eq. (2) can be rewritten as

$$
\begin{gathered}
I(x, y, z)=a_{\mathrm{R}}^{2}+\left|a_{\mathrm{O}}(x, y)\right|^{2}+2 a_{\mathrm{R}}\left|a_{\mathrm{O}}(x, y)\right| \cos \left(\varphi_{\mathrm{OF}}-\varphi_{\mathrm{RF}}\right) \\
=a_{\mathrm{R}}^{2}+\left|a_{\mathrm{O}}(x, y)\right|^{2}+2 a_{\mathrm{R}}\left|a_{\mathrm{O}}(x, y)\right| \cos \{2 k[L+z(x, y)]\} .
\end{gathered}
$$

Note $k=2 \pi / \lambda_{\mathrm{F}}$ is the wave vector for the LD with SMI effect, where $\lambda_{\mathrm{F}}=c / v_{\mathrm{F}}$ is the light wavelength with optical feedback. The OB surface topology $[z(x, y)]$ included in the phase difference $\varphi_{\mathrm{OF}}-\varphi_{\mathrm{RF}}$ can be reconstructed from the interference pattern $I(x, y, z)$.

\subsection{Theoretical Result for the Flat Surface}

If the measured surface is a flat one and perpendicular to the optical axis or $z$-axis, the term $L+z(x, y)$ will be a constant and no interfering fringes can be obtained. When the flat surface has a tilt angle with respect to the optical axis, and, thus, creates an intersecting angle $\theta$ between the $\mathrm{O}$ and $\mathrm{R}$ beams, the phase difference becomes

$\varphi_{\mathrm{OF}}-\varphi_{\mathrm{RF}}=2 k[L+z(x, y)]=2 k[L+y \sin (\theta / 2)]$.

Substituting $a_{R}=a_{O}(x, y)=1, L=20 \mathrm{~cm}$ into Eqs. (3) and (7), the interferogram for $\theta=10 \mathrm{deg}$ is obtained and shown in Fig. 2(a). They are a group of parallel fringes with same interval. The interval between two adjacent bright lines is $\Delta y=\lambda_{\mathrm{F}} / 2 \sin (\theta / 2)=2.32 \mu \mathrm{m}$. Within each fringe, the intensity changes from dark to bright gradually. The details can be seen from Fig. 2(b) for the selected two fringes. The features of such interference pattern can also be seen from Fig. 2(c) that is the waveform for the intensity distribution along $y$-direction with $x=0$. Clearly, the fringe is a saw-tooth like form.

\subsection{Theoretical Results for the Spherical Surface}

If the measured object is a spherical surface with a radius $R$, on the condition of paraxial approximation, the phase difference is $\varphi_{\mathrm{OF}}-\varphi_{\mathrm{RF}}=2 k\left[L-\left(x^{2}+y^{2}\right) / 2 R\right]$ for a concave surface and $\varphi_{\mathrm{OF}}-\varphi_{\mathrm{RF}}=2 k\left[L+\left(x^{2}+y^{2}\right) / 2 R\right]$ for a convex surface, and $L$ is the distance between the facet of LD and the vertex of the spherical surface.

Supposing $a_{\mathrm{R}}=a_{O}(x, y)=1, L=20 \mathrm{~cm}, R=10 \mathrm{~cm}$, and ignore the constant term, the interfering intensity is simplified from Eq. (7) and expressed as

$I(x, y)=\cos \left\{2 k\left[L \pm\left(x^{2}+y^{2}\right) / 2 R\right]\right\}$,

where "+" and "-" are related to the convex and concave surface, respectively. The corresponding interferogram for the concave and convex surface can be calculated and shown in Figs. 3 and 4, respectively. From these two figures, we can see the interferogram with concentric rings, which become denser from the central to the outside. But in the central area, the interfering field is different for both cases. We can find some ripple in the fringe shape, clearly 


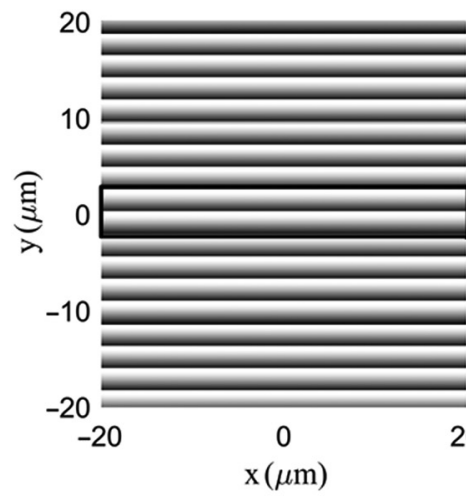

(a)

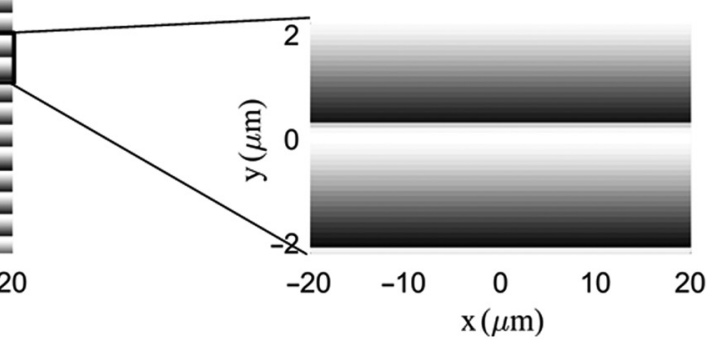

(b)

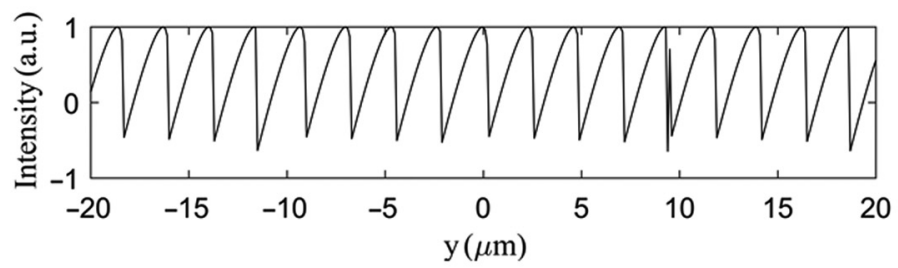

(c)

Fig. 2 (a) Interferogram in the $x-y$ plane, (b) detailed interferogram in the $x-y$ plane, and (c) intensity distribution with $x=0$.

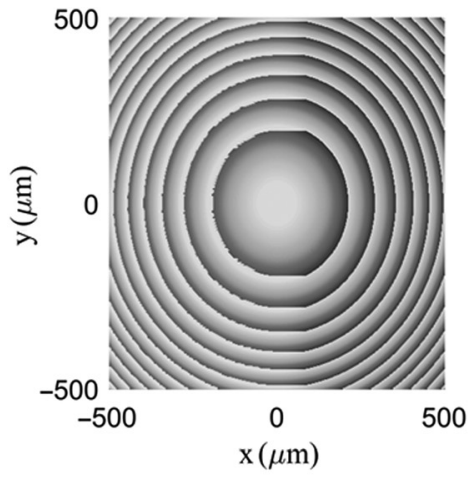

(a)

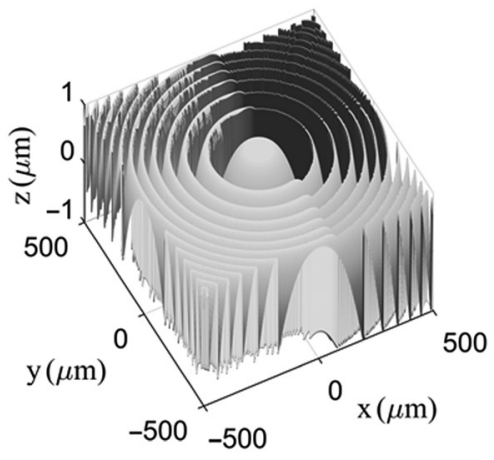

(b)

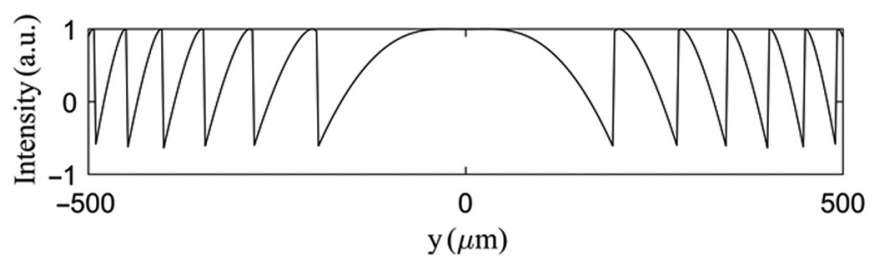

(c)

Fig. 3 For the concave surface: (a) interferogram in the $x-y$ plane, (b) intensity distribution in the $x-y$ plane, and (c) intensity with $x=0$.

due to the speckle pattern caused by the superposition of the random phases of the beams. ${ }^{19}$

In the two figures, when the phase difference $\varphi_{\mathrm{OF}}-$ $\varphi_{\mathrm{RF}}=2 j \pi$ where $j$ is an integer, the bright fringes appear and the dark fringes are corresponding to $\varphi_{\mathrm{OF}}-\varphi_{\mathrm{RF}}=(2 j+1) \pi$. So we can conclude that the radius of the bright rings is $r^{2}=2 R L-j R \lambda_{\mathrm{F}}$ and $r^{2}=j R \lambda_{\mathrm{F}}-2 R L$ for the concave and convex surfaces, respectively. For the concave surface, with the increase of the fringe order $j$, the radius decreases. But for the convex one, the changing trend is the opposite.

\subsection{Theoretical Result for the Surface with Arbitrary Topography}

Suppose the measured surface as an arbitrary surface with a radius of curvature satisfying the functional distribution 


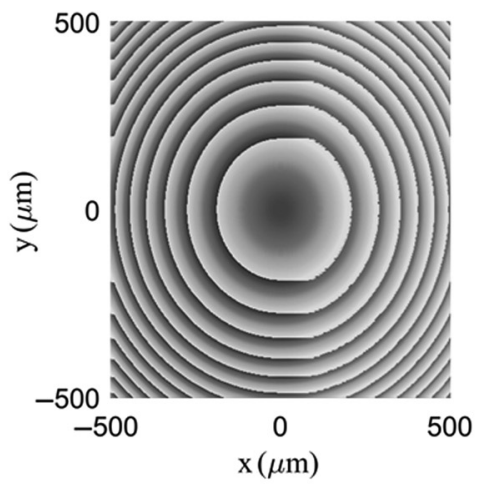

(a)

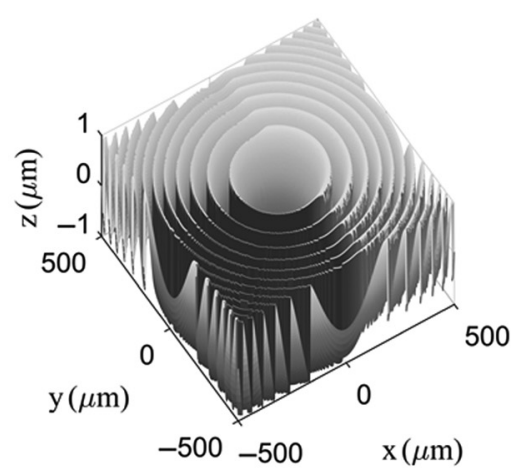

(b)

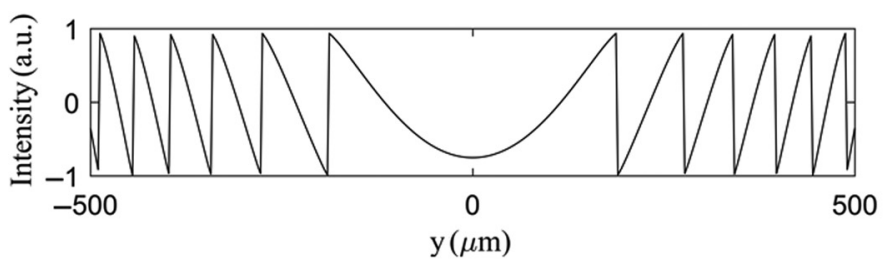

(c)

Fig. 4 For the convex surface: (a) interferogram in the $x-y$ plane, (b) intensity distribution in the $x-y$ plane, and (c) intensity with $x=0$.

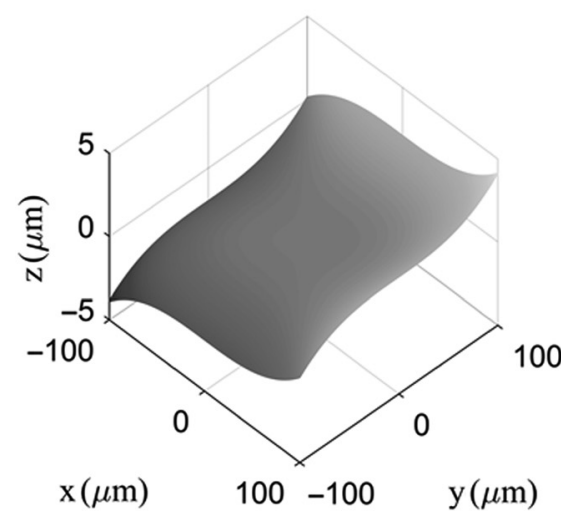

(a)

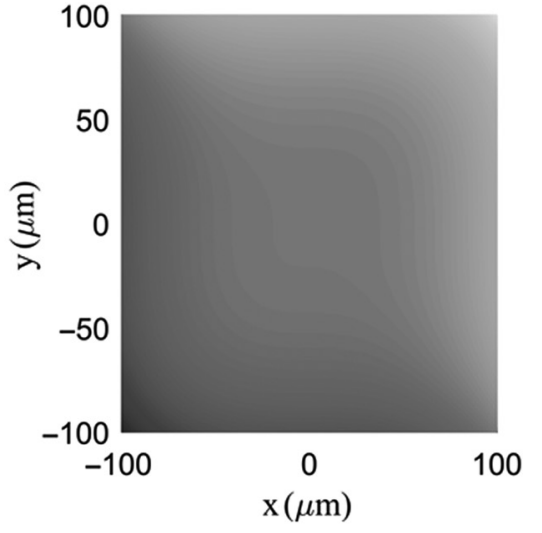

(b)

Fig. 5 For the asymmetrical curve surface: (a) surface topography and (b) contour plot.

$$
\begin{aligned}
z(x, y)= & 2 \times 10^{-6} \times x^{3}+2 \times 10^{-6} \times y^{3}+10^{-6} \times x^{2} \\
& +10^{-5} \times y^{2}+5 \times 10^{-5} \times x+3 \times 10^{-5} \times y
\end{aligned}
$$

The interfering patterns can also be calculated. According to the above distribution, the surface topography is plotted in Fig. 5. It is an asymmetrical curved surface. The surface can be flat, concave, or convex. At the same time, the contour lines are shown in Fig. 5(b). From Fig. 5(b), the asymmetry can be observed clearly.

Substitute Eq. (10), $a_{\mathrm{R}}=a_{\mathrm{O}}(x, y)=1$, and $L=20 \mathrm{~cm}$ into Eqs. (3) and (7), the interfering field can be calculated in Fig. 6. The interferogram is asymmetrical. The fringes are sparse in the center and get denser in the outside. The shape is very much like the contour plot in Fig. 5(b).

\section{Reconstruction of the Microsurface}

Given an intensity distribution of an interference pattern $I(x, y)$, we can reconstruct the surface of an object through Fresnel transformation. ${ }^{18}$ The transformation is represented as

$$
\begin{aligned}
\Gamma(\xi, \eta)= & \frac{i}{\lambda_{0} d} \exp \left(-i \frac{2 \pi d}{\lambda_{0}}\right) \exp \left[-i \frac{\pi}{\lambda_{0} d}\left(\xi^{2}+\eta^{2}\right)\right] \\
& \times \int_{-\infty}^{\infty} \int_{-\infty}^{\infty} u_{\mathrm{R}}^{*}(x, y) I(x, y) \exp \left[-i \frac{\pi}{\lambda_{0} d}\left(x^{2}+y^{2}\right)\right] \\
& \times \exp \left[i \frac{2 \pi}{\lambda_{0} d}(x \xi+y \eta)\right] \mathrm{d} x \mathrm{~d} y,
\end{aligned}
$$

where $d$ stands for the distance between the reconstruction plane and the CCD and $d=L$. 


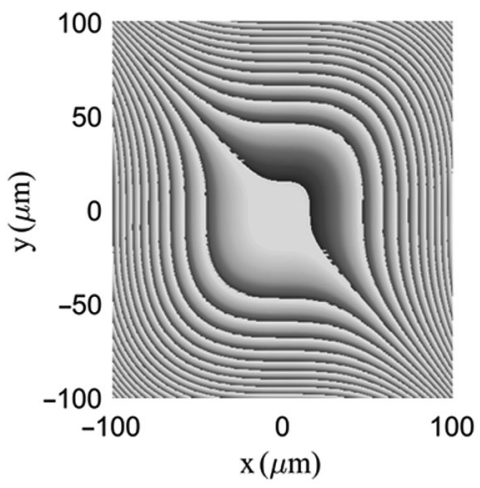

(a)

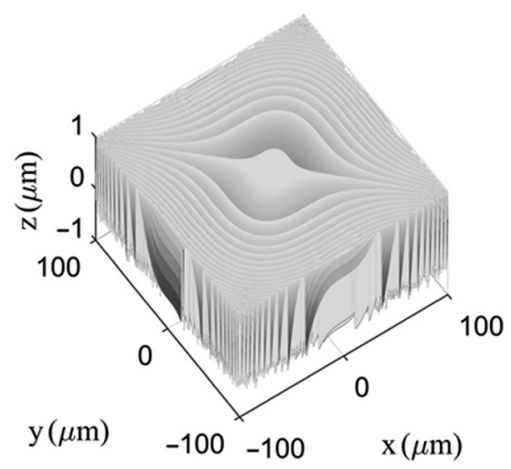

(b)

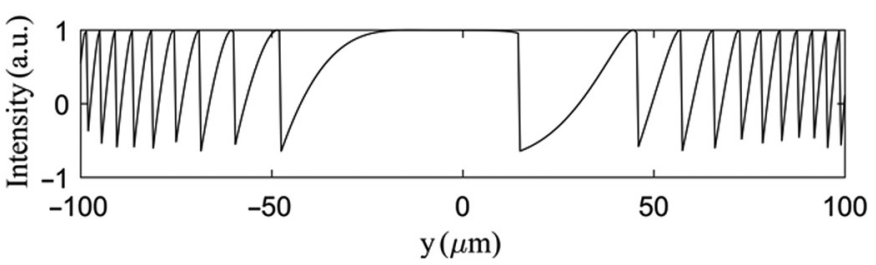

(c)

Fig. 6 For the asymmetrical surface: (a) interferogram in the $x-y$ plane, (b) intensity distribution in the $x-y$ plane, and (c) intensity with $x=0$.

From Eq. (11), the intensity and phase of the reconstructed surface can be calculated by $I(\xi, \eta)=|\Gamma(\xi, \eta)|^{2}$, $\varphi(\xi, \eta)=\arctan \frac{\operatorname{Im}[\Gamma(\xi, \eta)]}{\operatorname{Re}[\Gamma(\xi, \eta)]}$, where Re and Im denote the real part and the imaginary part, respectively. The phase is calculated directly by subtracting the reference and object beam phases modulo $2 \pi$ from the digital hologram. ${ }^{18}$ Using the convex surface described in Sec. 2.2 as an example, substitute the intensity distribution $I(x, y)$ described in Eq. (9) and $u_{\mathrm{R}}^{*}(x, y)=1, L=d=10 \mathrm{~cm}$, $R=10 \mathrm{~cm}$, and $\lambda_{0}=0.405 \mu \mathrm{m}$ into Eq. (11). Then, by calculating the phase of the object beam, the profile topology $z(x, y)$ can be reconstructed. The calculation in MATLAB ${ }^{\circledR}$ 2016 takes only several seconds. The original object surface

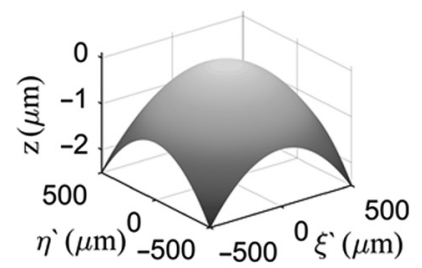

(a)

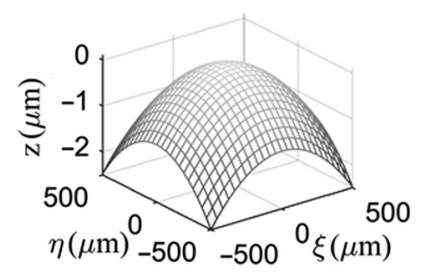

(c)

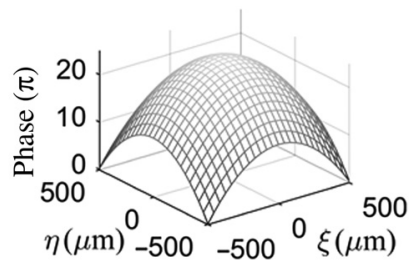

(b)

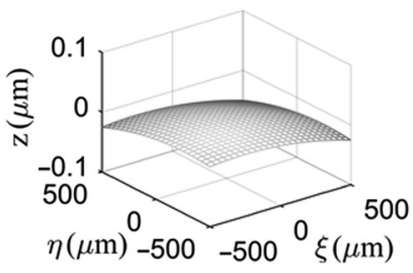

(d)
Fig. 7 (a) Convex surface, (b) unwrapped reconstructed phase $\varphi(\xi, \eta)$, (c) reconstructed surface, and (d) reconstruction error. is plot in Fig. 7(a). Figures 7(b) and 7(c) show the unwrapped object beam phase and the reconstructed surface, respectively. It can be seen that the 3-D surface can be reconstructed successfully. The reconstruction error shown in Fig. 7(d) is well-controlled within $0.05 \mu \mathrm{m}$.

\section{Conclusions}

The existing SMI-based imaging all is based on point-bypoint scanning, which is time-consuming. In this paper, we present a beam-expanded, SMI configuration for microsurface topography. First, the related theory and simulation are presented for describing the interference pattern obtained at CCD plane for different objects, and then the algorithm for reconstructing object profiles is developed using Fresnel transformation. The current work shows a potential configuration with compact structure and interference resolution for achieving microsurface measurement.

\section{Acknowledgments}

This work was supported by the Foundation Research Project of Henan Province (No. 152300410023) and the National Natural Science Foundation of China (No. 11104251).

\section{References}

1. R. Jones and C. Wykes, Holographic and Speckle Interferometry, Cambridge Studies in Modern Optics, Vol. 6, 2nd ed., Cambridge University Press, Cambridge (1989).

2. D. Guo and M. Wang, "Self-mixing interferometry based on a doublemodulation technique for absolute distance measurement," Appl. Opt. 46(9), 1486-1491 (2007).

3. M. Wang and G. Lai, "Displacement measurement based on Fourier transform method with external laser cavity modulation," Rev. Sci. Instrum. 72, 3440-3445 (2001).

4. S. Donati, M. Norgia, and G. Giuliani "Self-mixing differential vibrometer based on electronic channel subtraction," Opt. Lett. 45(28), 72647268 (2006). 
5. Y. Yu, J. Xi, and J. F. Chicharo, "Measuring the feedback parameter of a semiconductor laser with external optical," Opt. Express 19(10), 95829593 (2011).

6. R. Juškaitis, N. P. Rea, and T. Wilson, "Semiconductor laser confocal microscopy," Appl. Opt. 33(4), 578-584 (1994).

7. C. Lu, J. Wang, and K. Deng, "Imaging and profiling surface microstructures with noninterferometric confocal laser feedback," Appl. Phys. Lett. 66, 2022-2024 (1995).

8. V. J. Cemine et al., "High-contrast microscopy of semiconductor and metal sites in integrated circuits by detection of optical feedback," Opt. Lett. 29(21), 2479-2481 (2004).

9. C. M. Blanca et al., "Localizing defects on circuits using high-resolution OFT," Opt. Photonics News 17(12), 30 (2006).

10. E. Gagnon and J. F. Rivest, "Laser range imaging using the self-mixing effect in a laser diode," IEEE Trans. Instrum. Meas. 48(3), 693-699 (1999).

11. T. Bosch et al., "Three-dimensional object construction using a selfmixing type scanning laser range finder," IEEE Trans. Instrum Meas. 47(5), 1326-1329 (1998).

12. K. Bertling et al., "Simple electrical modulation scheme for laser feedback imaging," IEEE Sens. J. 16(7), 1937-1942 (2016).

13. E. Lacot, R. Day, and F. Stoeckel, "Coherent laser detection by frequency-shifted optical feedback," Phys. Rev. A 64(4), 043815 (2001)

14. O. Jacquin et al., "Acousto-optic laser optical feedback imaging," Opt. Lett. 37(13), 2514-2516 (2012).

15. H. S. Lui et al., "Terahertz inverse synthetic aperture radar imaging using self-mixing interferometry with a quantum cascade laser," Opt. Lett. 39(9), 2629-2632 (2014).

16. W. Glastre et al., "Demonstration of a plenoptic microscope based on laser optical feedback imaging," Opt. Express 21(6), 7294-7303 (2013).

17. W. Glastre et al., "Synthetic aperture laser optical feedback imaging using a translational scanning with galvanometric mirrors," J. Opt. Soc. Am. A 29(8), 1639-1647 (2012).

18. U. Schnars and W. Jüptner, Digital Holography, Springer-Verlag, Berlin, Heidelberg (2005)

19. A. A. Siddiqui et al., "All analog processing of speckle affected selfmixing interferometric signals," IEEE Sens. J. 17(18), 5892-5899 (2017).
Xiaohong Sun is a professor at Zhengzhou University. She received her PhD in optics from the University of Science and Technology of China in 2003. She is the author of more than 50 journal papers. Her current research interests include new type optical sensing material, holography, and optoelectronic systems.

Han Wang received his BE degree from Beijing University of Posts and Telecommunications, China, in 2010 and his ME degree from Zhengzhou University, China, in 2015 . He is currently a PhD candidate at the School of Electrical Computer and Telecommunications Engineering, University of Wollongong, Australia. His research interests include semiconductor lasers with optical feedback and their applications on sensing and instrumentations, displacement measurement, and holography.

Bin Liu received his BE degree from Beijing Jiaotong University, Beijing, China, in 2011 and his ME degree from the Institute of Microelectronics, Chinese Academy of Sciences, Beijing, China, in 2014 , both in electronic engineering. He is currently pursuing his $\mathrm{PhD}$ at the School of Electrical Computer and Telecommunications Engineering, University of Wollongong. His main research interests include laser optical feedback interferometry and its applications.

Yanguang Yu received her $\mathrm{BE}$ and $\mathrm{PhD}$ degrees from Huazhong University of Science and Technology, China, in 1986 and Harbin Institute of Technology, China, in 2000, respectively. She is an associate professor at the University of Wollongong, Australia. Her current research interests include optoelectronic systems, three-dimensional profile measurement, and telecommunication systems. 